\title{
Microhardness of binary near-equiatomic Ti-Ni alloys after severe cold rolling and post-deformation annealing
}

\author{
K. Inaekyan ${ }^{\mathrm{a} 1}$, V. Brailovski ${ }^{1}$, S. Prokoshkin ${ }^{2}$, A. Korotitskiy $^{2}$ and A. Chernavina ${ }^{2}$ \\ ${ }^{1}$ Ecole de Technologie Superieure, 1100, Notre-Dame Street West, Montreal, H3C 1K3, Canada \\ ${ }^{2}$ Moscow Institute of Steel and Alloys, 4, Leninsky prosp., Moscow 119049, Russia
}

\begin{abstract}
Comparative HV-microhardness and TEM studies of Ti-50.0at\%Ni and $50.26 \mathrm{at} \% \mathrm{Ni}$ alloys subjected to cold-rolling ( $\mathrm{e}=0.3,1$ and 1.72$)$ and post-deformation annealing at $100-700^{\circ} \mathrm{C}$ ( 1 hour) are presented. Based on the TEM-measured grain size data for Ti-50.0 at $\% \mathrm{Ni}$ alloy as a function of an annealing temperature $\left(\mathrm{T}_{\mathrm{PDA}}\right)$ higher than $250^{\circ} \mathrm{C}$, it was possible to evaluate the grain size (d) of the near-equiatomic Ti-Ni alloys at $\mathrm{T}_{\mathrm{PDA}}<250^{\circ} \mathrm{C}$, using exponential extrapolation distribution of the $d-T_{P D A}$ data. It was shown that below a critical grain size $\left(d_{c}=10 \mathrm{~nm}\right)$, the smaller the grain size (as a result of the decrease in annealing temperature), the lower the microhardness. This softening phenomena can be described, with good correlation between the approximation and experimental data, by the normal-abnormal Hall-Petch transition caused by the influence of the intercrystalline regions and by the melting temperature grain-size dependence.
\end{abstract}

\section{Introduction}

Binary near-equiatomic Ti-Ni alloys demonstrate the best combination of shape memory, corrosion, wear and fatigue resistance properties among all of the known shape memory alloys (SMA). Recently, it was found that thermomechanical processing consisting of severe plastic deformation (SPD) and post-deformation annealing (PDA) allows creation of a nanocrystalline structure in Ti-Ni SMA, which results in a significant improvement of their functional properties [1-3]. In a series of works, the stability of the amorphous/nanocrystalline structure formed by severe cold rolling (CR) was studied during room-temperature aging [4], and a peculiar dome-shape evolution of the alloys' microhardness (HV) as a function of the aging time has been pointed out. It is known that nanocrystalline metallic alloys do not follow the classical Hall-Petch behaviour and that below a certain grain size, softening instead of hardening is observed $[5,6]$. In this work, in the efforts to compare these phenomena, the microhardness of $\mathrm{Ti}-50.26 \% \mathrm{Ni}$ samples subjected to severe $\mathrm{CR}$ and annealing to form nanocrystalline, submicrocrystalline and recrystallized austenite structures was measured and analysis of the experimental data was performed using two generic models to describe the abnormal Hall-Petch behaviour of the nanocrystalline materials: model of the intercrystalline regions and model of the melting temperature grain-size dependence [7-9].

\section{Experimental obesrvations}

A Ti-50.26at\%Ni alloy wire $(\varnothing 1 \mathrm{~mm}$ ) was subjected to $\mathrm{CR}$ with logarithmic thickness reduction of $\mathrm{e}=0.3,1$ and 1.72. Microhardness (Vickers) measurements were performed using the INSTRON Wilson Tukon 2100 (500 g) on CR and annealed $\left(100-700^{\circ} \mathrm{C}, 1\right.$ hour) samples (Fig. 1a). It can be observed that for moderately coldworked alloy $(\mathrm{e}=0.3)$, an increase in annealing temperature leads to a continuous material softening, whereas for severely CR alloys ( $\mathrm{e}=1$ and 1.72), an increase in annealing temperature up to $250-300^{\circ} \mathrm{C}$ leads to a significant microhardness increase, followed by its drastic decrease, when these temperatures are exceeded.

For comparison, the DSC-measured transformation heat flow on the same samples is plotted as a function of the annealing temperature (Fig. 1b). It can be observed that for severely cold-worked samples (e $=1$ and 1.72), that up to annealing at $250^{\circ} \mathrm{C}$, the higher the annealing temperature, the higher the exothermic heat flow. When annealing temperature exceeds $325^{\circ} \mathrm{C}$, heat flow changes sign from positive (exothermic) to

a e-mail: karine.inaekyan.1@ens.etsmtl.ca

This is an Open Access article distributed under the terms of the Creative Commons Attribution-Noncommercial License (http://creativecommons.org/licenses/by-nc/3.0/), which permits unrestricted use, distribution, and reproduction in any noncommercial medium, provided the original work is properly cited. 
negative (endothermic), while being always decreasing and endothermic for moderately CR samples. The phenomena of the initial increase in heat flow can be explained by a strongly heterogeneous structure of the cold-rolled and annealed samples [1] with different start temperatures and progression rates of two coinciding transformations: a) crystallization of the amorphous phase and b) growth of the existing nanocrystals. Furthermore, it was shown [10] that in severely CR alloys with mixed amorphous-nanocrystalline structure, the dominant processes during annealing at relatively low temperatures are nucleation (i.e., nanocrystallization of amorphous phase) and grain growth, whereas in moderately CR-alloys, these are stress relaxation and recovery followed by polygonization. Which is why, for severely CR alloys ( $\mathrm{e}=1$ and 1.72), after annealing at temperatures below $325^{\circ} \mathrm{C}$, exothermic heat flow related to nucleation and grain growth phenomena is observed in the temperature range from 345 to $380^{\circ} \mathrm{C}$, whereas after annealing at higher temperatures (and at any temperature, for moderately $\mathrm{CR}$ alloys, $\mathrm{e}=0.3$ ), endothermic heat flow due to reverse martensitic transformation is measured in the temperature range from 80 to $175^{\circ} \mathrm{C}$.

Based on this analysis, it seems that both the $\mathrm{HV}\left(\mathrm{T}_{\mathrm{PDA}}\right)$ and $\Delta \mathrm{H}\left(\mathrm{T}_{\mathrm{PDA}}\right)$ behaviour are related to the same changes in the material microstructure and these changes can be attributed to the grain size variations. Evidently, the mechanical properties of metallic alloys are structure sensitive and depend therefore on all of the structural parameters, such as grain and subgrain size, phases in presence, structural defects, precipitates, impurities, etc. Grain size alone thus cannot be considered as the only parameter governing the mechanical properties of metallic alloys, and this is especially true for Ti-Ni shape memory alloys undergoing thermoelastic martensitic phase transformations. In this work, however, the main emphasis will be on the grain size as a major structural parameter.

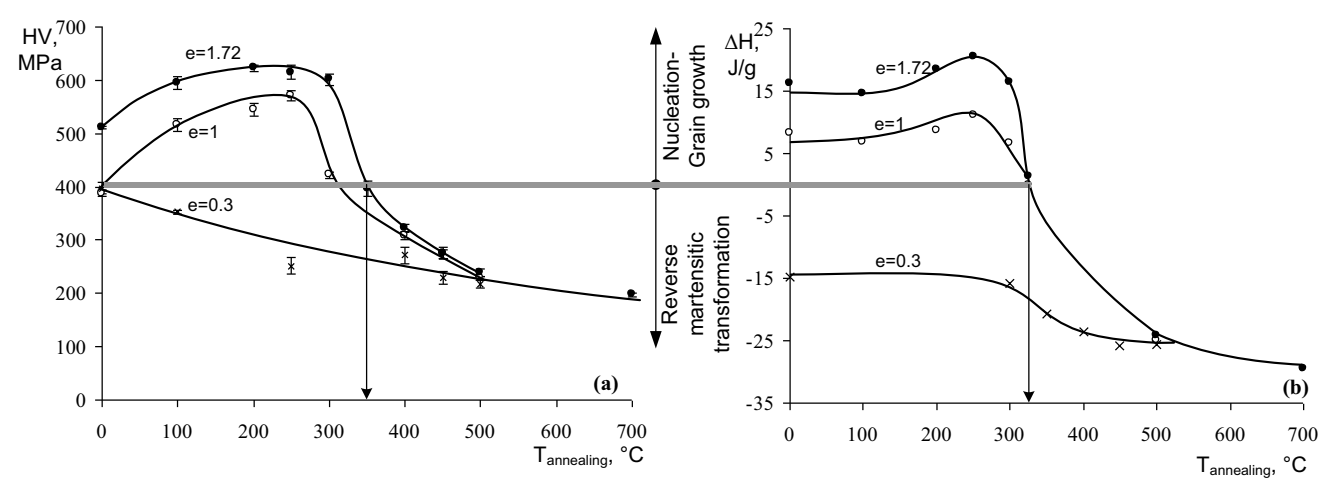

Fig. 1. HV microhardness of Ti-50.26\%Ni alloy after CR $(0.3-1.72)$ and annealing at $100-500^{\circ} \mathrm{C}(1 \mathrm{~h})(\mathrm{a})$ and the heat flow effect (b)

It is known that SPD of binary Ti-Ni alloys by means of severe cold rolling $(\mathrm{e}=1.9)$ leads to the formation of a mixed (approximately 70/30) nanocrystalline and amorphous structure: 2-8 nm (average grain size $5 \mathrm{~nm}$ ) B2-austenite nanograins are dispersed in the amorphous matrix [11]. In Fig.2a, the HV(T $\left.\mathrm{T}_{\text {PDA }}\right)$ plot obtained for Ti-50.26at $\% \mathrm{Ni}\left(\mathrm{e}=1.72\right.$, same as in Fig. 1a) is superposed on the $\mathrm{d}\left(\mathrm{T}_{\mathrm{PDA}}\right)$ plot for Ti-50.0at $\% \mathrm{Ni}(\mathrm{e}=$ 1.9) and a combined $\mathrm{HV}(\mathrm{d})$ graph is plotted in Fig.2b. Note that the grain size measurements were performed by $\mathrm{TEM}$ at $\mathrm{T}_{\mathrm{PDA}}>250^{\circ} \mathrm{C}[11]$. Since the structure of the severely cold-worked alloy is a mixed nanocrystalline and amorphous structure, it is reasonable to suppose that the size of a representative structural parameter varies in the triangular grey-shaded zone shown on Fig. 2a,b. This zone is upper-bound by a line representing an interpolation of the grain size at $\mathrm{T}_{\mathrm{PDA}}=250^{\circ} \mathrm{C}(12.5 \mathrm{~nm})$ to $\mathrm{T}_{\mathrm{PDA}}=0{ }^{\circ} \mathrm{C}\left(\mathrm{d}_{\min }=5 \mathrm{~nm}\right)$ and it is lower-bound by a line representing an extrapolation of all the measured $\mathrm{d}-\mathrm{T}_{\mathrm{PDA}}$ data at $\mathrm{T}_{\mathrm{PDA}}>250^{\circ} \mathrm{C}$ to $\mathrm{T}_{\mathrm{PDA}}=0^{\circ} \mathrm{C}\left(\mathrm{d}_{0}=0.19 \mathrm{~nm}\right)$.
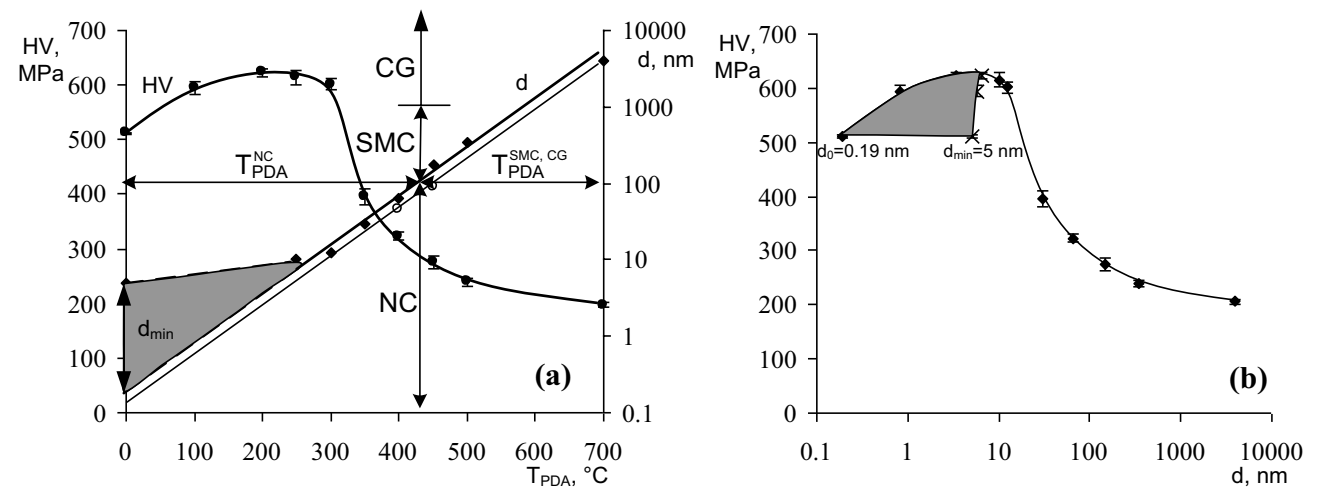

Fig. 2. (a) Grain size in Ti-50.0at $\% \mathrm{Ni}$ alloy after SPD (e=1.9) and $\mathrm{HV}$ in Ti-50.26\%Ni alloy after SPD (e=1.72) as a function of the annealing temperature (1h) [11]; NC- nanocrystalline, SMC - submicrocrystalline, CG - coarse-grained; •-Ti$50.0 \% \mathrm{Ni}$, ०-Ti-50.26\%Ni; (b) combined "HV-d" graph for $\mathrm{d}_{0}=0.19$ and $\mathrm{d}_{\min }=5 \mathrm{~nm}$. 
Note: Applying the linear approximation of $\operatorname{Ln}(d)-T_{P D A}$ to the scarce grain size data existing for Ti$50.26 \% \mathrm{Ni}$ alloy (Fig. 2a, white circles) gives $d_{0}=0.14 \mathrm{~nm}$ at $T_{P D A}=0^{\circ} \mathrm{C}$. The difference between 0.14 and $0.19 \mathrm{~nm}$ is about $30 \%$. For further analysis, the experimental data obtained for Ti-50.0\%Ni alloy (Fig. 2a, black quadrates) will be used until a larger quantity of TEM data becomes available for Ti-50.26\% Ni alloy.

\section{Hall-petch relationship}

\subsection{Introduction}

An increase in strain resistance (yield stress, hardness) of crystals with decreasing grain size $d$ is commonly described by the empirical Hall-Petch relationship:

$$
\sigma(d)=\sigma_{o}{ }^{\prime}+k_{d} \cdot d^{-1 / 2},
$$

where $\sigma_{o}{ }^{\prime}=\sigma(d \rightarrow \infty)$ is the yield stress of the defect-free single crystal, and $k_{d}{ }^{\prime}$ is the positive material constant (Hall-Petch slope) dependent on the grain boundaries' resistance to dislocation movement. The normal $\mathrm{H}-\mathrm{P}$ relationship is generally explained on the basis of a dislocation pile-up at grain boundaries. However, below a critical grain size (around $10 \mathrm{~nm}$ for metals), no dislocation activity is observed and plastic deformation occurs mostly by grain boundary sliding $[5,6,8,9,12]$ with increasing influence of: (a) interface regions mobility $[8,9$, $13,14]$ and (b) melting temperature decrease [15-18], both resulting in material softening if grains become sufficiently small.

Interface regions: Palumbo et al. [14] have shown that for grain sizes below $30 \mathrm{~nm}$, the triple junction volume fraction manifests a greater grain size dependence than the grain boundary volume fraction, and for grain sizes under $3 \mathrm{~nm}$, the fraction of triple junctions become greater than that of grain boundaries. The authors explain the softening effect caused by the interface regions by their structure-atomic spacing, which deviate from the equilibrium state of the regular lattice [13]. That is why the grain boundaries and triple junctions are usually assumed as an amorphous structure $[8,9]$.

Melting temperature: another peculiarity of nanocrystalline materials is the widely observed grain-size dependence of the melting temperature $\left(\mathrm{T}_{\mathrm{m}}\right)$ [15-18]. The main theoretical basis of the melting temperature decrease with significant grain refinement is based on the size-dependent amplitude of atomic thermal vibrations in terms of the Lindemann criterion on melting (at melting temperature, the expansible vibration amplitude in all metals is the same, which results in material softening [19]).

In this work, these two phenomena influencing deformation mechanisms of NC materials will be modeled for binary Ti-Ni alloys and the numerical results compared with the experimental data.

\subsection{Model of material softening based on the influence of interface regions}

\subsubsection{Generalities}

Based on the results of $[8,9]$, an NC material is assumed to be a composite material consisting of crystallite, grain boundary surface, triple line junction, and quadratic node. The grain shape is approximated by a regular rectangle (d) with grain boundary width (w). The three-dimensional grain model is illustrated in Fig. 3a. The grain component volume is calculated as follows (Fig. 3b):

$$
\begin{aligned}
& f_{c r}=\frac{(d-w)^{3}}{d^{3}} \text { (crystallite), } f_{g b}=\frac{6(d-w)^{2}(w / 2)}{d^{3}} \text { (grain boundary surface), } \\
& f_{t j}=\frac{12(d-w)(w / 2)^{2}}{d^{3}} \text { (triple line junction), and } f_{q n}=\frac{w^{3}}{d^{3}} \text { (quadratic node). }
\end{aligned}
$$

The main assumption of the model is: all of the intercrystalline components have amorphous structure, so their volume can be estimated as $1-f_{c r}$. Based on [19-21], the grain boundary thickness for the coarsegrained and nanocrystalline materials is approximately $1 \mathrm{~nm}(w \approx 3 b$, where $b-$ Burgers vector). 


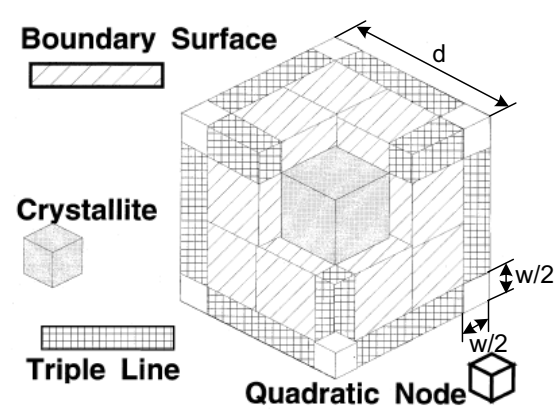

(a)

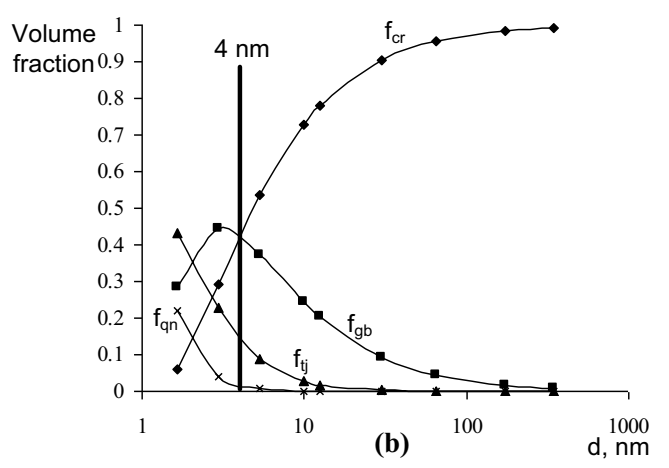

(b)

Fig. 3. (a) Grain representation in $3 \mathrm{D}$ composite model [8]; (b) volume fraction of the crystallite $\left(\mathrm{f}_{\mathrm{cr}}\right)$, grain boundaries $\left(\mathrm{f}_{\mathrm{gb}}\right)$, triple junctions $\left(\mathrm{f}_{\mathrm{tj}}\right)$ and quadratic nodes $\left(\mathrm{f}_{\mathrm{qn}}\right)$ as a function of the grain size

Fig. $3 \mathrm{~b}$ clearly shows that the grain size of approximately $4 \mathrm{~nm}$ represents a grain dimension when intercrystalline components becomes dominant [14]. To superpose the influence of each component, the total strength (or Young's modulus or hardness) can be calculated as follows:

$$
H V=H V_{c r} f_{c r}+H V_{g b} f_{g b}+H V_{t j} f_{t j}+H V_{q n} f_{q n}
$$

Based on the facts that the Hall-Petch relationship is valid for crystalline regions and that contribution to hardness of the intercrystalline regions is proportional to their fractions, and that the critical grain size ( $d_{c}$ ) limits the zone of validity of the normal Hall-Petch relationship, equation (2) can be transformed as follows [8]: If $d \geq d_{c}$, the Hall-Petch relationship is valid:

$$
H V=\left(H V_{o}^{i r}+k_{h p} d^{-1 / 2}\right) f_{c r}+H V_{i c}\left(1-f_{c r}\right)
$$

If $d \leq d_{c}$, the Hall-Petch relationship is not valid (dislocation pile-up fails, so the crystallite would not be hardened):

$$
H V=\left(H V_{o}^{i r}+k_{h p} d_{c r}^{-1 / 2}\right) f_{c r}+H V_{i c}\left(1-f_{c r}\right)
$$

\subsection{Model of material softening based on the melting temperature grain-size dependence}

\subsubsection{Generalities}

To describe the material softening of nanocrystalline materials, the grain-size dependence of the material melting temperature $\left(\mathrm{T}_{\mathrm{m}}\right)$ is considered [15-18]. Following Jiang et al [7], when $\mathrm{d}<100 \mathrm{~nm}, \sigma_{o}{ }^{\prime}$ and $k_{d}{ }^{\prime}(1)$ become temperature-dependent:

$$
\sigma(d)=\sigma_{o}{ }^{\prime}+\left[k_{t}{ }^{\prime}+k_{d}{ }^{\prime} d^{-1 / 2}\right] \exp \left[\frac{T_{m}(d)}{2 T_{d}}\right],
$$

where $\sigma_{o}{ }^{\prime}=\sigma_{o}+k_{t}{ }^{\prime} \exp \left[\frac{T_{m}(d)}{2 T_{d}}\right] ; k_{d}{ }^{\prime}=k_{d}{ }^{\prime \prime} \exp \left[\frac{T_{m}(d)}{2 T_{d}}\right]$,

The value of $\sigma_{o}$ is the temperature-independent initial strength; $k_{t}{ }^{\prime} \exp \left[\frac{T_{m}(d)}{2 T_{d}}\right]$ is the temperaturedependent strength component, where $k_{t}{ }^{\prime}$ is a constant, $T_{m}(d)$ is the grain size-dependent melting temperature and $T_{d}$ is the testing temperature; $k_{d}{ }^{\prime}$ is the grain size-dependent Hall-Petch constant depicting the migration resistance of dislocations; and $k_{d} "$ is a constant.

Using the relation: $T_{m}(d)=T_{m 0} \exp \left[\frac{-2 S_{m} /(3 R)}{d / d_{o}-1}\right]$, where $S_{m}=H_{m} / T_{m 0}$ and $S_{m}, H_{m}$ and $T_{m 0}$ are the bulk melting entropy, enthalpy and temperature, respectively, the modified Hall-Petch relationship describing, in a single formulation, both the hardening and the softening behaviour, can be expressed as follows [7]:

$$
\sigma(d)=\sigma_{o}{ }^{\prime}+\left[k_{t}{ }^{\prime}+k_{d}{ }^{\prime} d^{-1 / 2}\right] \exp \left[\frac{T_{m 0}}{2 T_{d}} \exp \left(-\frac{2 H_{m} /(3 R)}{T_{m 0}\left(d / d_{o}-1\right)}\right)\right]
$$


Considering that for small $\mathrm{x}, e^{-x}=1-x$, equation (4) can be rewritten:

or

$$
\begin{aligned}
& \sigma(d)=\sigma_{o}{ }^{\prime}+\left[k_{t}{ }^{\prime}+k_{d}{ }^{\prime} d^{-1 / 2}\right] \exp \left[\frac{T_{m 0}}{2 T_{d}}-\frac{\alpha}{d / d_{0}-1}\right] \\
& \sigma(d)=\sigma_{o}{ }^{\prime}+\left[k_{t}+k_{d} d^{-1 / 2}\right] \exp \left[-\frac{\alpha}{d / d_{0}-1}\right]
\end{aligned}
$$

where $k_{t}=k_{t}^{\prime} \cdot \exp \left[\frac{T_{m o}}{2 T_{d}}\right], k_{d}=k_{d}{ }^{\prime \prime} \cdot \exp \left[\frac{T_{m o}}{2 T_{d}}\right], \alpha=\frac{H_{m} /(3 R)}{T_{d}}$; for TiNi alloy $H_{m}=32.5 \mathrm{~kJ} / \mathrm{mol}$ [22].

Note that $d_{0}$ represents a crystal with almost all of its atoms located on its surface. According to [23], for nanocrystalline materials, $d_{o} \cong 6 h(h-$ is the mean diameter of the atoms in the solution, for elements and compounds - atomic or molecular diameter). For $\mathrm{Ni}, h=0.2754 \mathrm{~nm}$ [7], so $\mathrm{d}_{0}=1.65 \mathrm{~nm}$, which represent a minimum crystal size. The smallest nanograin, which can be treated with model (8), corresponds to that with at least a half of the atoms located inside it: $\mathrm{d}_{\min }{ }^{\mathrm{NC}}=2 \mathrm{~d}_{0}=3.3 \mathrm{~nm}$.

\subsection{Analysis of the experimental results for Ti-50.26\% Ni alloy}

Based on the previous discussion, $\mathrm{d}_{0}=0.19 \mathrm{~nm}$ (the value obtained from the extrapolation of the TEM data for "Ln(d)- $\mathrm{T}_{\text {PDA }}$ ") (Fig. 2b) will be replaced a minimum crystal size of $1.65 \mathrm{~nm}$ [7]. The values $\mathrm{d}_{0}=1.65 \mathrm{~nm}$ and $\mathrm{d}_{\min }=5 \mathrm{~nm}$ will be used for the calculations. Fig. 4 a presents the experimental data for $\mathrm{d}_{0}=1.65 \mathrm{~nm}$ and $\mathrm{d}_{\min }=$ $5 \mathrm{~nm}$ with approximation according to equations (3) and (4), where the coefficients $H V_{o}^{i r}, K_{h p}$ and $H V_{i c}$ were estimated by the least square method. Fig. 4 illustrates the results of analysis of the experimental data according to the (a) model of interface regions, and (b) model of melting temperature grain-size dependence. For comparison, the conventional Hall-Petch (1) graph is superposed on Fig.4a.

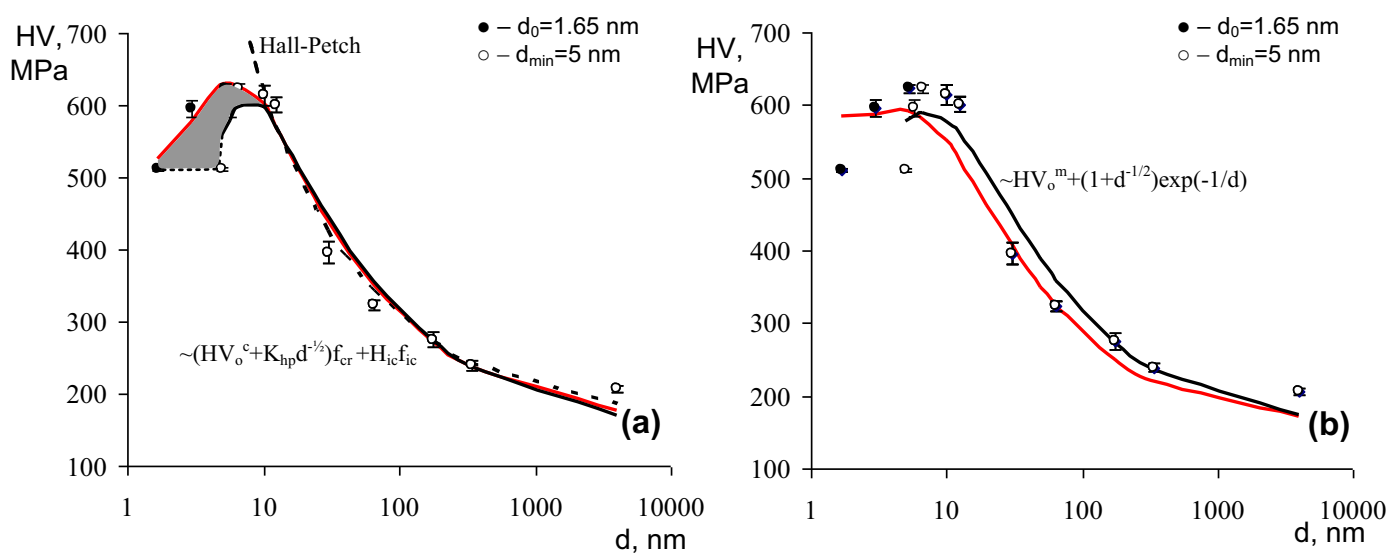

Fig. 4. Combined results of the normal-abnormal Hall-Petch analysis by (a) model of interface regions and the conventional Hall-Petch and (b) model of the melting temperature grain-size dependence

It can be observed that the modified H-P relationship (8) ("grain size-melting temperature" model) applied to the experimental data of Fig. $2 \mathrm{~b}$ leads to an appropriate correspondence between the experimental and calculated data for $\mathrm{d}>12.5 \mathrm{~nm}$ (Fig. 4b). But in the region between $\mathrm{d}_{0}\left(\mathrm{~d}_{\min }\right)$ and $12.5 \mathrm{~nm}$, equation (8) does not adequately describe the material softening. In the case of the "interface regions model", the approximation according to (3) and (4), (Fig. 4a) shows an appropriate correlation with the experimental results in the whole grain sizes range.

The approximation coefficients for both methods are collected in Table 1 and Table 2.The coefficients $k_{h p}$ and $k_{d}$ in both models are common Hall-Petch coefficients and are of the same order, but they are smaller than those found for polycrystalline $\mathrm{Ni}$ (5059 $\mathrm{MPa} \cdot \mathrm{nm}^{1 / 2}$ [24]). This difference could be associated with the difference in the mechanical behaviour of pure metals and intermetallics. The softening threshold grain size determined by both models $\mathrm{d}_{\mathrm{c}} \approx 10 \mathrm{~nm}$ represents a critical grain size for NC Ti-Ni alloys. This hypothesis corresponds to the observed softening of $\mathrm{NC}$ materials below a grain size of $10 \mathrm{~nm}[6,25]$. 
Table 1. Approximation parameters of the interface regions model

\begin{tabular}{|l|c|c|}
\hline Parameter & $\mathbf{d}_{\mathbf{0}}=\mathbf{1 . 6 5} \mathbf{~ n m}$ & $\mathbf{d}_{\mathbf{m i n}}=\mathbf{5} \mathbf{~ n m}$ \\
\hline$H V_{o}{ }^{i r}, \mathrm{MPa}$ & 153 & 143 \\
\hline$H V_{i c}, \mathrm{MPa}$ & 513 & 355 \\
\hline$k_{h p}, \mathrm{MPa} \cdot \mathrm{nm}^{1 / 2}$ & 1530 & 1719 \\
\hline
\end{tabular}

Table 2. Approximation parameters of the melting temperature grain-size dependence model

\begin{tabular}{|l|c|c|}
\hline Parameter & $\mathbf{d}_{\mathbf{0}}=\mathbf{1 . 6 5} \mathbf{~ n m}$ & $\mathbf{d}_{\mathbf{m i n}}=\mathbf{5} \mathbf{~ n m}$ \\
\hline$H V_{o}{ }^{m}, \mathrm{MPa}$ & 586 & 547 \\
\hline$k_{t}, \mathrm{MPa}$ & -434 & -398 \\
\hline$k_{d}, \mathrm{MPa} \cdot \mathrm{nm}^{1 / 2}$ & 1116 & 1500 \\
\hline$\alpha$ & \multicolumn{2}{|c|}{4.45} \\
\hline
\end{tabular}

\section{Remarks:}

Model of interface regions: in the case of $\mathrm{d}_{\min }=5 \mathrm{~nm}$, the $H V_{i c}=355 \mathrm{MPa}$ is significantly lower compared to experimentally measured in this study $511 \mathrm{MPa}$ (Table 1 and Fig. 1a), whereas the value of $H V_{o}{ }^{i r}$ correlates rather well with the $H V$ of the quenched alloy.

Model of the melting temperature grain-size dependence: contrary to the results of Zhao et al. [7], coefficient $k_{t}$ is negative to reflect a decrease in lattice thermal resistance.

Models comparison: In the $\mathrm{d}<\mathrm{d}_{\mathrm{c}}$ range, the alloy's softening is described better by the model of interface regions than by that of the melting temperature grain-size dependence (Fig. 4), which reflects stronger influence of the interface regions on the deformation of the studied nanocrystalline Ti-Ni alloy.

\section{Conclusions}

1. An abnormal Hall-Petch relationship is observed in Ti-Ni shape memory alloy with nanocrystalline structure obtained by severe plastic deformation and post-deformation annealing and the critical grain size for the normal-abnormal Hall-Petch relationship transition is around $10 \mathrm{~nm}$.

2. The alloy's softening is described better by the model of interface regions than by that of the melting temperature grain-size dependence, which reflect stronger influence of the interface regions on the deformation mechanisms of the studied nanocrystalline Ti-Ni alloys.

\section{Acknowledgements}

The authors would like to thank the Natural Sciences and Engineering Research Council of Canada and the Federal Agency for Education of the Russian Federation for their financial support.

\section{References}

[1]. V. Brailovski et al., Materials Transactions, 47(3), 795 (2006).

[2]. S.D. Prokoshkin et al., Materials Science and Engineering A, 481-482, 114 (2008).

[3]. V. Demers et al., Materials Science and Engineering A, 513-514, 185 (2009).

[4]. A.V. Korotitskiy et al., Materials Science Forum, Nanomaterials by Severe Plastic Deformation IV, 584-586, 1039 (2008).

[5]. K. Lu, M.L. Sui, Scripta Metallurgica et Materialia, 28, 1465 (1993).

[6]. C. Suryanarayana et al., Journal of Materials Research, 7(8), 2114 (1992).

[7]. M. Zhao, J.C. Li, Q. Jiang, Journal of Alloys and Compounds, 361, 160 (2003).

[8]. H.S. Kim, Scripta Materialia, 39(8), 1057 (1998).

[9]. X. Qing, G. Xingming, International Journal of Solids and Structures, 43, 7793 (2006).

[10]. K. Inaekyan et al., Journal of Alloys and Compounds, 473, 71 (2009).

[11]. K.E. Inaekyan et al., Materials Science Forum: 503-504, 597 (2005).

[12]. T.G. Nieh, J. Wadsworth, Scripta Metallurgica et Materialia, 25(4), 955 (1991).

[13]. H. Gleiter, Acta Materialia, 48, 1 (2000).

[14]. G. Palumbo, S.J. Thorpe, K.T. Aust, Scripta Metallurgica et Materialia, 24(7), 1347 (1990).

[15]. M. Takagi, J. Phys. Soc. Jpn., 9, 359 (1954).

[16]. Z. Zhang, J.C. Li, Q. Jiang, Journal of Physics D: Applied Physics, 33(20), 2653 (2000).

[17]. A.N. Goldstein, Applied Physics, A 62, 33 (1996).

[18]. K.N. Shrivastava, Nano Letters, 2(5), 519 (2002). 
[19]. H. Gleiter, Progress in Materials Science, 33(4), 223 (1989).

[20]. D.G. Brandon et al., Acta Materialia, 12(7), 812 (1964).

[21]. J. Budai, W. Gandig, S.L. Sass, Philosophical Magazine A: Physics of Condensed Matter, Structure, Defects and Mechanical Properties, $40(6), 759$ (1979).

[22]. H.C. Yi, J.J. Moore, Journal of Materials Science, 24, 3449 (1989).

[23]. Q. Jiang, H.X. Shi, M. Zhao, Journal of Chemical Physics, 111(5), 2176 (1999).

[24]. A.W. Thompson, Acta Metallurgica, 23(11), 1337 (1975).

[25]. H. Conrad, J. Narayan, Scripta Materialia, 42, 1025 (2000). 